Results Hepatic packing with drainage and blood pressure control were done. Patient received Low Molecular Weight Heparin and Aspirin and improved after 13 days without bleeding. Conclusions Spontaneous hepatic rupture is a rare, life-threatening presentation of APAS that warrants timely diagnosis and effective control of haemorrhage to prevent maternal and fetal mortality. Early initiation of anticoagulation is both safe and effective in preventing APAS complications. Aspirin should be initiated to prevent future thrombosis and pregnancy loss.

\section{STUDY OF CLINICAL UTILITY OF ANTIBODIES TO PHOSPHATIDYLSERINE/PROTHROMBIN COMPLEX IN INDIAN PATIENTS}

A Ganapati*, R Goel, J Kabeerdoss, M Gowri, J Mathew, D Danda. Christian Medical College - Vellore, Rheumatology, Vellore, India

\subsection{6/lupus-2017-000215.62}

Background and aims To explore utility of Antibodies to Phosphatidylserine/Prothrombin Complex (aPSPT), in Indian Anti phospholipid Syndrome (APS) patients.

Methods Data of 372 subjects whose sera was tested for aPSPT by ELISA (AESUKU,GERMANY), was retrospectively analysed. Performance of various APLs was compared using non-parametric tests. To ascertain additional utility of aPSPT, patients with suspected APS were categorised into 2 groups $i$. e. i) those with any one of criteria antibodies positive ii) those with any one APL positivity including anti- PSPT positivity.

Results In 46 patients of APS, 38 (82.60\%) had thrombotic events (21 arterial , 26 venous) and 11 ( 23.91\%) had obstetric events. Lupus anticoagulant was present in 37/46 (80.43\%) , anticardiolipin antibody in 19/46 (41.33\%), aPSPT in $17 / 46$ (36.95\%), anti- $\beta 2$ glycoprotein1 ( $\beta 2 \mathrm{GP} 1)$ IgG and IgM in 13/ $46(28.26 \%)$ and $7 / 46(15.21 \%)$ patients of APS respectively. aPSPT tested positive in $9 / 60(15 \%)$ patients with seronegative APS (SNAPS). aPSPT did not differ from other criteria APLs in any clinical parameter except compared to those with IgM B2GP-1 positivity. aPSPT was numerically more sensitive and specific than IgM B2GP1 for thrombotic, non-criteria events but less sensitive for detecting obstetric events (Table 2). Inclusion of aPSPT test in patients with suspected APS increased

Abstract 62 Tabel 1 Showing the categorization of Study subjects into patients and controls

\begin{tabular}{|l|l|l|}
\hline Type of Subjects & Number & Sub-categorization \\
\hline Total Number of Subjects & 372 & Female-331 \\
\cline { 2 - 3 } & & Male-41 \\
\hline $\begin{array}{l}\text { Definite APS (satisfying revised } \\
\text { Sapporo criteria) }\end{array}$ & 46 & Primary-20 \\
\cline { 3 - 3 } & & Secondary-26 \\
\hline Controls & 326 & $\begin{array}{l}\text { Patients with seronegative } \\
\text { APS/SNAPS)-60 }\end{array}$ \\
\cline { 3 - 3 } & & $\begin{array}{l}\text { Connective Tissue Disease } \\
\text { controls-146 }\end{array}$ \\
\cline { 3 - 3 } & & Obstetric Controls-107 \\
\cline { 3 - 3 } & & Healthy Controls-13 \\
\hline
\end{tabular}

Abstract 62 Tabel 2 Comparision of sensitivity and specificity of aPSPT and IgM anti- $\beta 2$ GP1 in definite APS patients

\begin{tabular}{|l|l|l|l|l|}
\hline Definite APS patients & \multicolumn{2}{|l|}{ Anti PSPT positive } & \multicolumn{2}{l|}{ IgM Anti-B2GP antibody positive } \\
\hline & Sensitivity & Specificity & Sensitivity & Specificity \\
\hline Obstetric Events & $27.3 \%$ & $38.5 \%$ & $45.5 \%$ & $11.5 \%$ \\
\hline Arterial Events & $38.1 \%$ & $32 \%$ & $9.5 \%$ & $24 \%$ \\
\hline Venous Events & $38.5 \%$ & $30 \%$ & $11.5 \%$ & $25 \%$ \\
\hline $\begin{array}{l}\text { Non criteria } \\
\text { manifestations }\end{array}$ & $50 \%$ & $29.4 \%$ & $16.7 \%$ & $17.6 \%$ \\
\hline
\end{tabular}

sensitivity marginally but with concomitant decrease in specificity.

Conclusions In our study, aPSPT was third most common antibody in APS patients, displaying higher sensitivity and specificity in comparison to IgM anti- $\beta 2$ GP1 for all APS manifestations except obstetric ones. SNAPS patients may test positive for aPSPT, thus endorsing its inclusion in classification criteria of APS.

\section{RITUXIMAB: IN THE MANAGEMENT OF ANTIPHOSPHOLIPID ANTIBODIES ASSOCIATED CHOREA IN A CHILD WITH LUPUS}

${ }^{1} \mathrm{~A}$ Jindal ${ }^{*},{ }^{1} \mathrm{P}$ Vignesh, ${ }^{1} \mathrm{~A}$ Gupta, ${ }^{2} \mathrm{~J}$ Ahluwalia, ${ }^{1} \mathrm{D}$ Suri, ${ }^{1} \mathrm{~A}$ Rawat, ${ }^{1} \mathrm{~S}$ Singh. ${ }^{1} \mathrm{PGIMER}$, Paediatrics, chandigarh, India; ${ }^{2}$ PGIMER, haematology, chandigarh, India

\subsection{6/lupus-2017-000215.63}

Background and aims Neuropsychiatric manifestations are noted in 50.94\% of children with lupus. Chorea, a nonthrombotic complication associated with antiphospholipid antibodies (APLA), is seen in 4\% cases.

Aim- To describe the clinical course of a child with lupus who had chorea on follow up and was treated with rituximab Methods A 10 year old girl presented with spastic quadriparesis, fever, weight loss, alopecia, oral ulcers, anaemia and thrombocytopenia. Antinuclear antibody was $4+$ diffuse pattern; and anti-dsDNA titres were $220.25 \mathrm{IU} / \mathrm{ml}$ (normal $<55 \mathrm{IU} / \mathrm{ml}$ ). T2 weighted magnetic resonance imaging (MRI) brain and spine showed multiple foci of increased signal intensity in bilateral corona radiata and centrum semiovale. She also had positive lupus anticoagulant and elevated anticardiolipin antibody titres. She received steroids, azathioprine, anticoagulants and aspirin, and showed remarkable improvement. Five years later, she was admitted with multiple joint pains and foot drop related to mononeuritis multiplex. She developed seizures and MRI brain showed diffuse cerebral atrophy with T2/FLAIR white matter hyperintensities. At 18 years of age, she presented with chorea and MRI showed periventricular T2/FLAIR hyperintensities. She responded to intravenous pulses of methylprednisolone and cyclophosphamide. Three years later, she again had similar choreiform movements with aggressive behaviour, which did not respond to haloperidol, steroids and warfarin. At this point of time, she was administered 3 doses of rituximab $\left(500 \mathrm{mg} / \mathrm{m}^{2}\right.$ at one weekly interval). 
Results She showed complete recovery after rituximab.

Conclusions Rituximab can be considered as an effective treatment modality in APLA related chorea where conventional measures have failed.

\section{DYSBIOSIS AND GUT BARRIER DYSFUNCTION IN ANTIPHOSPHOLIPID SYNDROME AS REVEALED BY IGA- SEQ PROFILING}

$1,{ }^{2}$ WJ Kim* ${ }^{2}$ W Ruff, ${ }^{3} \mathrm{C}$ Aguiar, ${ }^{2} \mathrm{~A}$ Yu, ${ }^{2} \mathrm{SM}$ Vieira, ${ }^{2} \mathrm{JA}$ Sterpka, ${ }^{4} \mathrm{~A}$ Goodman, ${ }^{3} \mathrm{D}$ Erkan, 2,5 MA Kriegel. 'Cheju Halla General Hospital, Laboratory Medicine, Jeju-si, Republic of Korea; ${ }^{2}$ Yale School of Medicine, Immunobiology, New Haven, USA; ${ }^{3}$ Hospital for Special Surgery, Medicine, NewYork, USA; ${ }^{4}$ Yale School of Medicine, Microbial Pathogenesis, New Haven, USA; ${ }^{5}$ Yale School of Medicine, Medicine, New Haven, USA

\subsection{6/lupus-2017-000215.64}

Background and aims The antiphospholipid syndrome (APS) is an autoimmune thrombophilic non-gut disorder with high mortality. Various pathogens have been associated with transient antiphospholipid antibody production. We hypothesised that members of the gut microbiota in APS patients could represent a chronic trigger and exhibit heightened adaptive immune responses to the microbiota. The purpose of this study was to explore gut barrier function and faecal IgAcoated microbial composition in APS patients.

Methods Stool from 15 APS patients, 5 non-autoimmune thrombotic states, and 12 normal donors (total of 17 controls) was collected. Faecal homogenates were analysed for the gut permeability marker calprotectin and, in parallel, stained stained with PE-conjugated anti-human IgA prior to cell sorting. Faecal DNA was isolated and PCR-amplified targeting the V4 region of the $16 \mathrm{~S}$ rRNA gene. Samples were sequenced on the Illumina MiSeq platform.

Results Faecal calprotectin and IgA-coated faecal bacterial levels were significantly higher in APS patients compared to controls $(\mathrm{p}<0.003 ; \mathrm{p}<0.05)$. LEfSe analysis of IgA+ fractions showed that the strongest IgA-coated genus is Blautia in APS.

Conclusions These data suggest gut barrier dysfunction and aberrant IgA coating of commensals in APS. Markedly enhanced bacterial IgA coating in several APS patients supports a stronger adaptive immune response to the microbiota. Increased IgA coating of Blautia might reflect altered gut homeostasis as a Blautia species was shown to be part of proinflammatory $\operatorname{IgA}+$ consortium in a recent study in IBD. To our knowledge, this study represents the first 16S rRNA profiling of IgA-coated gut commensals in patients with nongut autoimmunity.

\section{ANTIPHOSPHOLIPID SYNDROME LONG TERM FOLLOW UP: REAL LIFE EXPERIENCE OF A SINGLE CENTRE}

${ }^{1} \mathrm{RM}$ Serrano Morales*${ }^{*}{ }^{2} \mathrm{G}$ Pons-Estel, ${ }^{3} \mathrm{R}$ Quintana, ${ }^{2} \mathrm{G}$ Espinosa Garriga, ${ }^{4} \mathrm{R}$ Cervera Segura. 'Barcelona, Spain; ${ }^{2}$ Hospital Clínic, Autoimmune Diseases Unit, Barcelona, Spain; ${ }^{3}$ Hospital Provincial de Rosario, Reumatología, Rosario, Argentina; ${ }^{4}$ Hospital Clínic Barcelona, Autoimmune Diseases Unit, Barcelona, Spain

\subsection{6/lupus-2017-000215.65}

Background and aims The antiphospholipid syndrome (APS) has wide clinical and outcome spectrum overtime. The aim of this study was to assess the real life prevalence of morbi-mortality APS during 10 year-follow-up of a single referral centre.
Methods The cohort included 160 consecutive APS patients followed by the Autoimmune Diseases Unit of Hospital Clínic Barcelona from 2003 to 2013. Epidemiological, clinical, immunological and treatment features were analysed prospectively.

Results The cohort consisted of 126 (79\%) women and 34 (21\%) mens. Mean (SD) age at diagnosis was 39 (14) years. The diagnostic causes were thrombosis $(56.3 \%)$, obstetric morbidity (26.9\%) and both (16.9\%). 65\% were primary APS, $22.5 \%$ associated with systemic lupus erythematosus (SLE), $8.8 \%$ associated with lupus-like syndrome and $3.7 \%$ associated with other diseases. Fifty-five patients were lost to follow-up (3.4\% every year). In evolution, 10 primary APS patients were reclassified as SLE-associated APS and 2 patients developed catastrophic APS. Table 1 shows the frequencies of the main APS clinical manifestations at baseline and during the 10-year-follow-up. At diagnosis, 95\% received antithrombotic treatment: low dose antiaggregants (39.5\%), oral anticoagulants $(67.1 \%)$, heparin $(2.6 \%)$. During the study, $72.7 \%$ of recurrences were without antithrombotic treatment and $27.3 \%$ were despite it. Eleven major bleeding episodes occurred and 2 were fatal. The global mortality rate was $6.9 \%$ and $50 \%$ in catastrophic APS. Table 2 shows the main causes of death and Figure 1 is a Kaplan-Meier survival curve.

Conclusions This study shows long-term morbidity and mortality of a large APS patient cohort and exposed the real-life experience of a referral unit.

\section{ANTIPHOSPHOLIPID SYNDROME - CINICAL AND IMMUNOLOGICAL CORRELATIONS AND ATHEROSCLEROSIS}

N Stoilov*, V Boyadzhieva, R Rashkov. University hospital "St. Ivan Rilski", Clinic of Rheumatology, Sofia, Bulgaria

\subsection{6/lupus-2017-000215.66}

Background and aims In recent years, it is found that role in atherogenesis plays inflammatory component of immune response. In recent years found unarguably data for accelerated development of atherosclerotic plaques in patients with APS.

Our aim is to investigate the frequency of cardiovascular events and atherosclerosis in patients with primary and secondary antiphospholipid syndrome compared to healthy individuals and patients with systemic lupus erythematosus without antiphospholipid antibodies.

Methods We studied 99 patients with APS, 13 SLE and 32 healthy controls. They were tested for aPL antibodies (aCL, anti-b2gp1, anti-prothrombin), ANA-screen, ANA - profile and standart laboratory.

We examine Intima-Media thickness of carotit arteries and Calcium score of: a. coronaria sinister, a. anterior descendens sinister, a. circumflexa sinister, a. coronaria dexter, Aorta, Valvaorte to validate the atheroslerosis.

Results We found strong, statistically significant correlation between aCL antibodies and the presence of plaques in the left common carotid artery $(\mathrm{p}=0.041)$ and absent a correlation between aPL titers and presence of carotid plaques. In the group with APS, 33,3\% (14) establishes a positive calcium score of coronary artheries, $11.9 \%$ (5) positive for aorta, Aortic valve Absent deposits, In the control group positive calcium score is one person $(5.88 \%)$. 